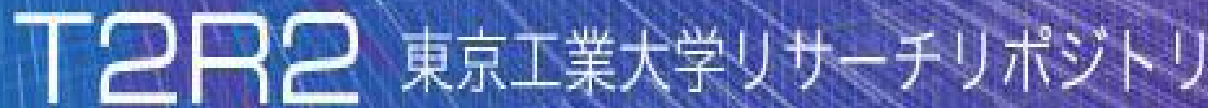

\section{Tokyo Tech Research Repository}

\section{論文 /著書情報 \\ Article /Book Information}

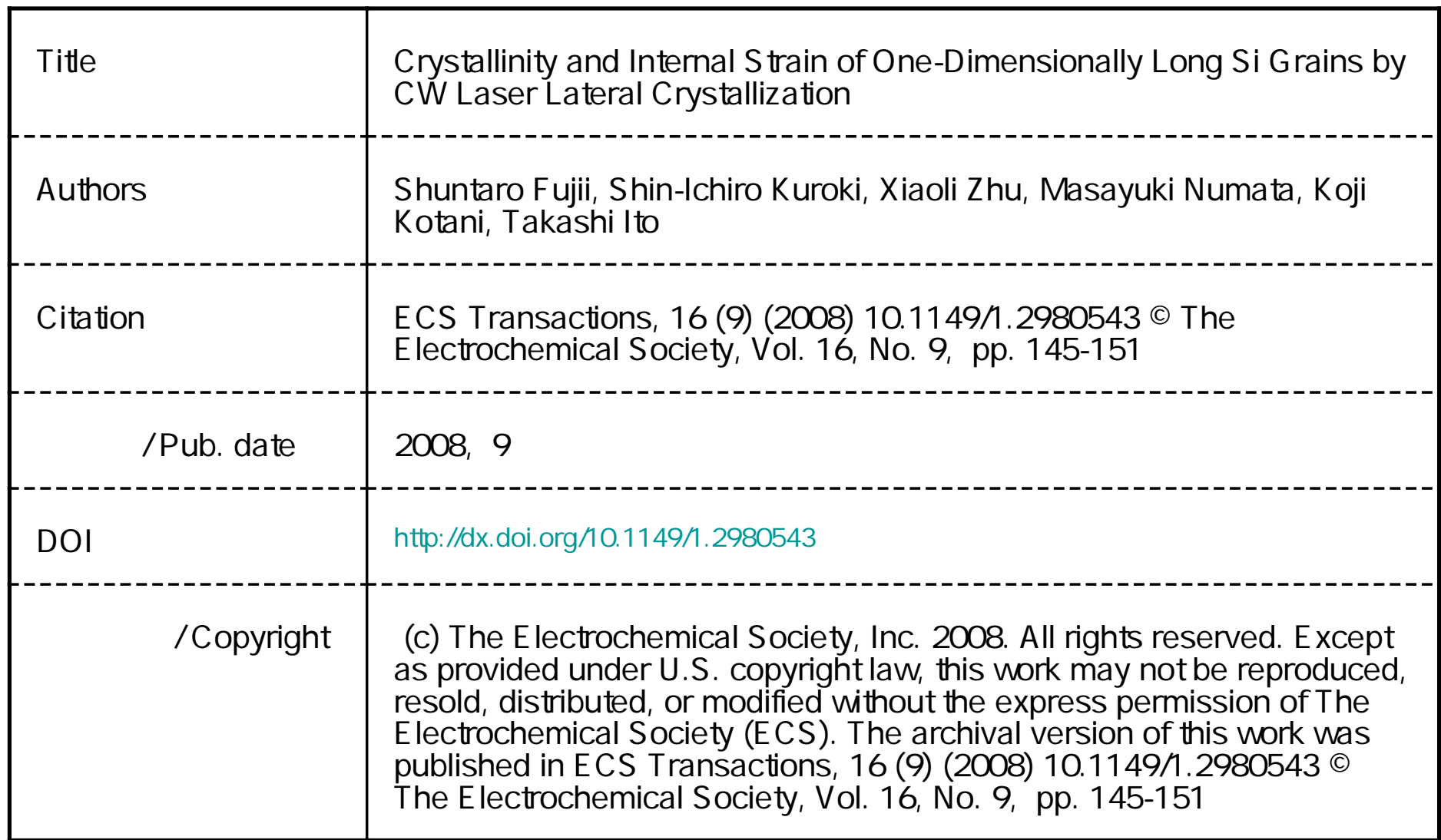




\title{
Crystallinity and Internal Strain of One-Dimensionally Long Si Grains by CW Laser Lateral Crystallization
}

\author{
Shuntaro Fujii, Shin-Ichiro Kuroki, Xiaoli Zhu, Masayuki Numata, \\ Koji Kotani, and Takashi Ito \\ Graduate School of Engineering, Tohoku University \\ 6-6-05 Aza-Aoba, Aramaki, Aobaku, Sendai, 980-8579, Japan \\ E-mail: \{sfujii, kuroki, tito\}@ecei.tohoku.ac.jp
}

Continuous-wave (CW) laser lateral crystallized silicon ( $\mathrm{Si})$ thin films were investigated. CW laser with a wavelength $\lambda$ of $532 \mathrm{~nm}$ was applied to crystallization. In the $\mathrm{CW}$ laser lateral crystallization (CLC), a gradual temperature slope in Si thin films was formed and one-dimensionally long grains with a typical length of $20 \mu \mathrm{m}$ were achieved. The most dominant grain growth direction was $<110>$ direction. $\sum 3,9$, and 11 boundaries were mainly observed between adjacent grains. It was found that crystallization was performed to form stable grain boundaries. It was also clarified that CLC poly-Si thin films have large tensile strain corresponding to $0.6 \%$ of single crystalline Si lattice in inplane direction. This strain was induced by the difference in thermal expansion coefficients between Si thin films and the buffer $\mathrm{SiO}_{2}$ films.

\section{Introduction}

High performance polycrystalline silicon (poly-Si) thin film transistors (TFTs) with high carrier mobility are expected for system-on-glass application. Well crystallized poly-Si thin films with large grains are indispensable to achieve high carrier mobility. Mobility in poly-Si TFTs using conventional excimer laser annealing (ELA) is degraded by grain boundary scattering (1). To reduce grain boundary scattering, one-dimensionally long grains are needed. Various crystallization methods to form one-dimensionally long grains have been proposed, such as phase-modulated ELA (2), sequential lateral solidification (SLS) (3), and continuous-wave (CW) laser lateral crystallization (CLC) $(4,5)$. It is thought that CLC is the most promising candidate to realize high performance TFTs with high carrier mobility because CLC can form the longest grains among them. For precise design of TFTs, the crystallinity should be clarified because carrier mobility greatly depends on surface orientation and channel direction (6). However, the crystallinity has not been clarified well. In addition, the strain in the CLC poly-Si films has not been clarified. It is well known that strain in the channel region can change carrier mobility because strain can modify the band structure and effective mass of Si (7). The strain in CLC poly-Si thin films also should been clarified.

In this paper, crystallinity and internal strain of CLC poly-Si thin films were investigated. Mechanisms of crystal growth of one-dimensionally long Si grains and induction of internal strain in CLC poly-Si thin films were discussed. Especially, the internal strain value of the CLC poly-Si thin films were quantitatively evaluated. 


\section{Experiments}

Figure 1 shows a schematic diagram of the sample structure for the CLC experiments. A buffer $\mathrm{SiO}_{2}$ film with a thickness of $1 \mu \mathrm{m}$ was deposited on a quartz substrate by plasma enhanced chemical vapor deposition (PECVD). An undoped amorphous Si (a-Si) film with a thickness of $150 \mathrm{~nm}$ was deposited by PECVD using $\mathrm{SiH}_{4}$ gas. After annealing in $\mathrm{N}_{2}$ ambient at $490^{\circ} \mathrm{C}$ for 20 min to reduce the hydrogen content in the a-Si thin films, the a-Si thin films were irradiated by diode pumped solid state (DPSS) CW laser with a wavelength $\lambda$ of $532 \mathrm{~nm}$ as shown in Fig. 2. The laser spot shape was ellipse, and the intensity profile was Gaussian shape with dimensions of $90 \times 20 \mu \mathrm{m}^{2}$ (full width at half maximum, FWHM). Crystallinity and internal strain in CLC poly-Si thin films were investigated by electron back-scattering diffraction pattern (EBSP) measurement, $\mathrm{X}$-ray diffraction (XRD) measurement, Raman spectroscopy, and transmission electron microscopy (TEM).

\begin{tabular}{|c|}
\hline $\begin{array}{c}\text { a-Si: } 150 \mathrm{~nm} \\
\text { (PECVD, undoped) }\end{array}$ \\
\hline $\begin{array}{c}\text { Buffer } \mathrm{SiO}_{2}: 1 \mu \mathrm{m} \\
\text { (PECVD) }\end{array}$ \\
\hline Quartz substrate \\
\hline
\end{tabular}

Fig. 1. Schematic diagram of the sample structure for CLC experiments.

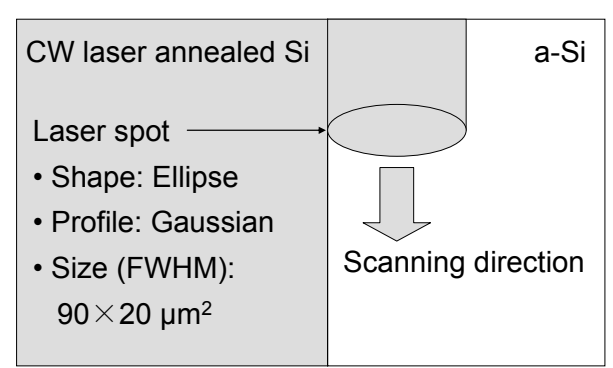

Fig. 2. Schematic image of CW laser spot on Si thin film in CLC experiments.

\section{Results and Discussion}

Figure 3(a) shows dependences of the structure of CW laser annealed Si thin films on laser power and scanning speed. At the laser power of $10 \mathrm{~W}$, lateral crystallization was

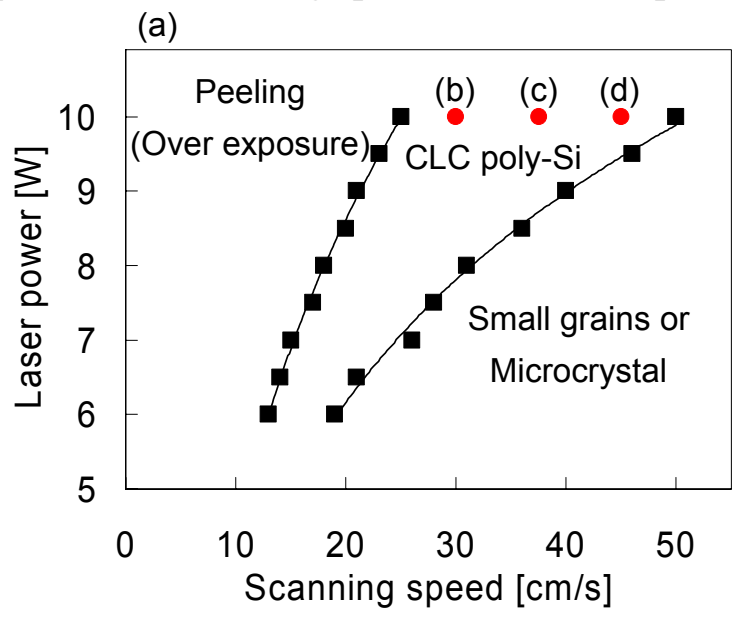

Fig. 3. (a) Dependences of the structure of CW laser annealed Si thin films on laser power and scanning speed. (b)-(d) Optical microscope images of CLC poly-Si thin films crystallized at the scanning speed of $30,37.5$, and $45 \mathrm{~cm} / \mathrm{s}$, respectively. Laser power was fixed at $10 \mathrm{~W}$.
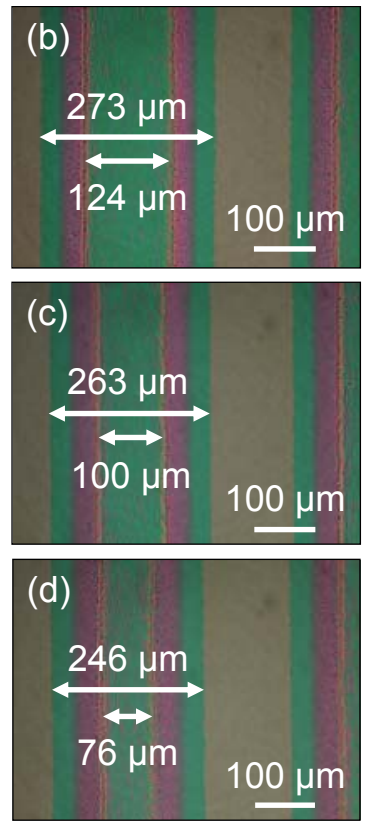
performed in the scanning speed range of from 25 to $50 \mathrm{~cm} / \mathrm{s}$. One-dimensionally long grains were formed in the center of $\mathrm{CW}$ laser annealed region. As increasing scanning speed with constant laser power, the CLC poly-Si region was decreased. Widths of CLC poly-Si region and total crystallized region formed by one scanning were shown in Fig. 3(b)-(d).

\section{Crystallinity of CLC poly-Si thin films}

Figure 4 shows normalized peak intensity of each surface orientation in XRD in-plane measurements as a function of CLC area ratio. The peak intensities were normalized by XRD intensities of Si powder. CLC area ratio was defined by an area ratio between CLC poly-Si area and total crystallized area. CLC area ratio was altered by CW laser scanning speed as shown in Fig. 3(b)-(d). Only the (220) peak intensity increased as CLC area ratio increased. It was found that the most dominant crystal growth direction was $<110>$ direction.

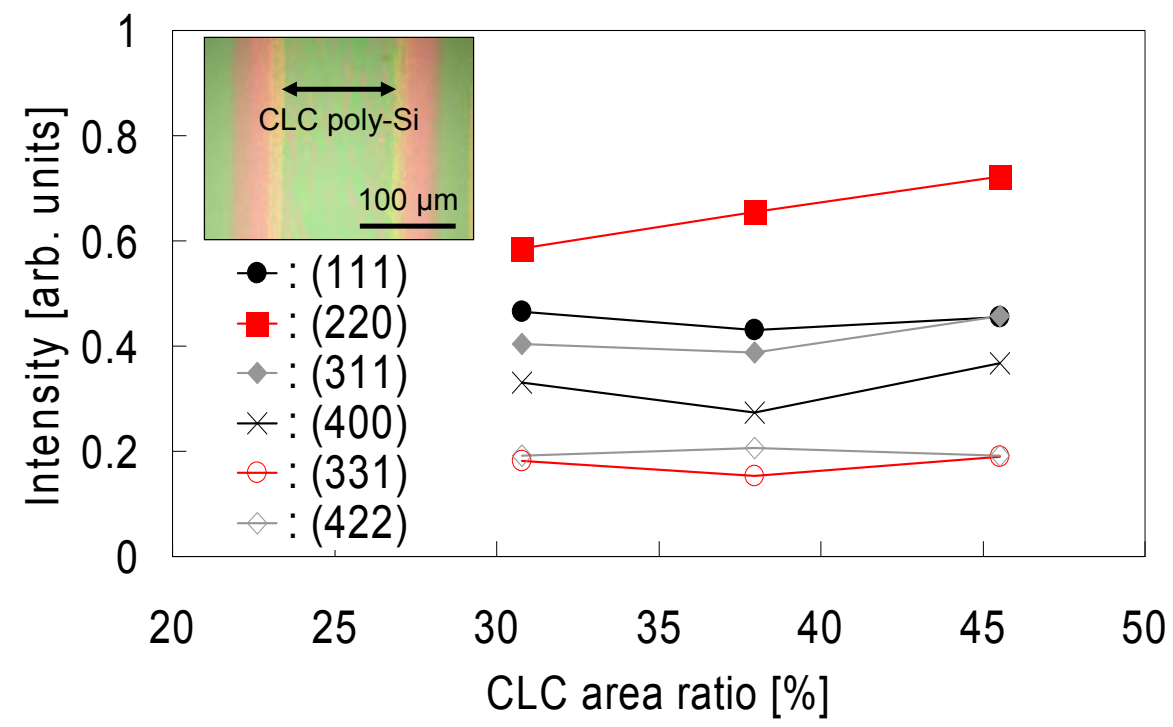

Fig. 4. Normalized peak intensity of each surface orientation in $\mathrm{XRD}$ in-plane measurements as a function of CLC area ratio.

Figure 5 shows the EBSP measurement results for the CLC poly-Si thin film. Laser power and scanning speed were $10 \mathrm{~W}$ and $30 \mathrm{~cm} / \mathrm{s}$, respectively. Figure 5(a) shows surface orientation of each grain in the CLC poly-Si thin film. Surface orientation of each grain in the CLC poly-Si thin film was not uniform. The typical grain size was $20 \times 2$ $\mu \mathrm{m}^{2}$. Because quartz substrates have low thermal conductivity, heat diffusion from $\mathrm{Si}$ thin films to quartz substrates was small, and a gradual temperature slope along the scanning direction was easily formed in the Si thin films. As a result, grains grew along $\mathrm{CW}$ laser scanning direction and one-dimensionally long grains were formed. Figure 5(b) shows grain boundaries in the CLC poly-Si thin film. $\sum 3,9$, and 11 boundaries were mainly observed between adjacent grains.

Crystal growth of one-dimensionally long grains in $\mathrm{Si}$ thin films by CLC was summarized in Fig. 6. Figure 6(a) shows a cross-sectional schematic image of crystal growth parallel to the $\mathrm{CW}$ laser scanning direction. Grains grow along the laser scanning direction and the interface between Si grain and melting Si has a tendency to take grow along $<110>$ direction. Figure 6(b) shows a cross-sectional schematic image of crystal 
growth perpendicular to the $\mathrm{CW}$ laser scanning direction. It has been reported that $\sum 3,9$, and 11 boundaries along $<110>$ direction axis are electrically inactive because they have small interfacial energy, bond-length and bond-angle distortions (8). It is thought that grains rotate around the $<110>$ direction axis to make stable grain boundaries.
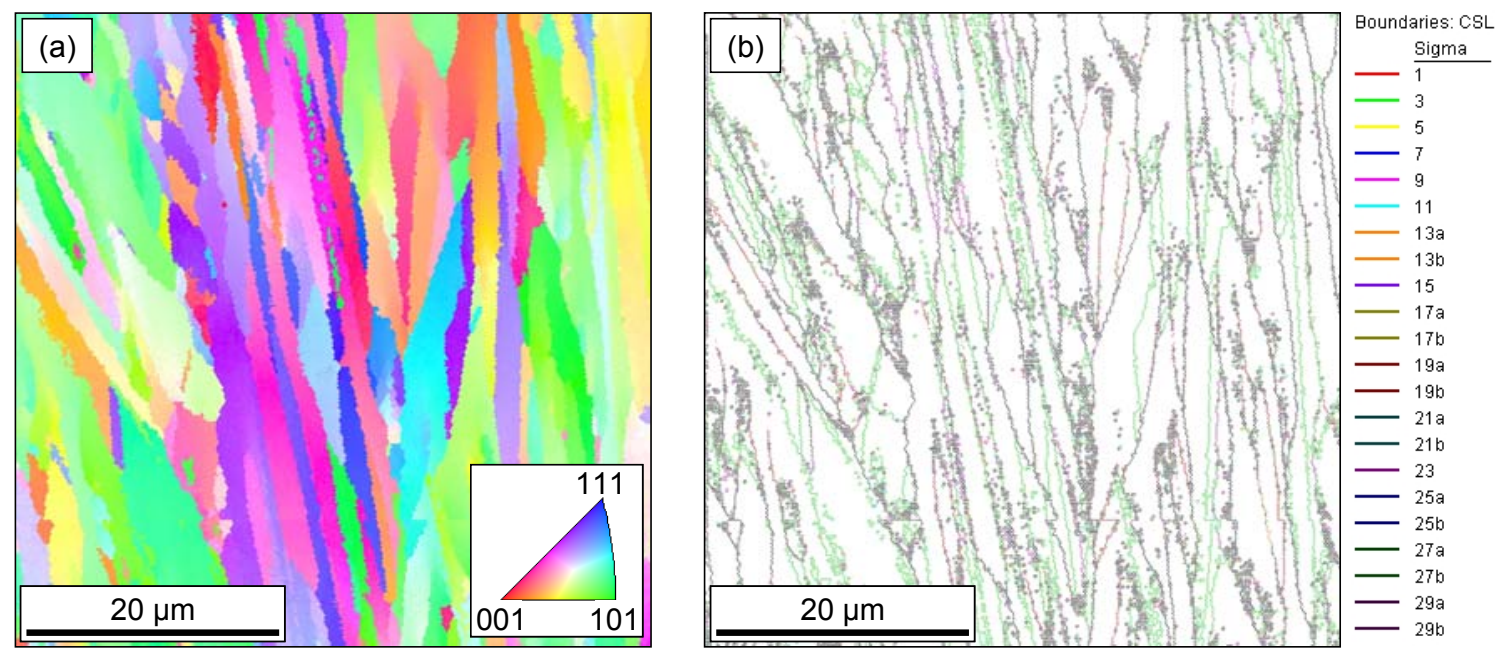

Fig. 5. EBSP measurement results for the CLC poly-Si thin film. (a) and (b) show surface orientation of each grain and grain boundaries in the CLC poly-Si thin film, respectively.

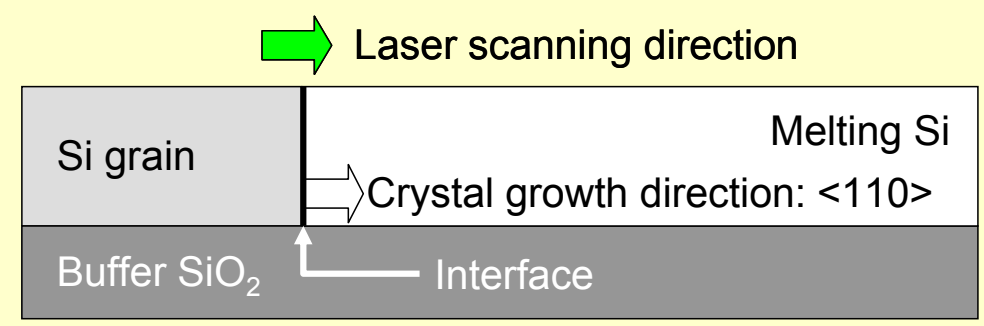

(a)

\begin{tabular}{|l|l|} 
Stable interface \\
$(\Sigma 3,9$, and 11)
\end{tabular}

(b)

Fig. 6. Cross-sectional schematic image of crystal growth of one-dimensionally long grains in a CLC poly-Si thin film. (a) and (b) are the images parallel and perpendicular to the laser scanning direction, respectively.

\section{$\underline{\text { Internal Strain of CLC poly-Si thin films }}$}

Figure 7 shows Raman spectra of a CLC poly-Si thin film and a single crystal Si. The diameter of the laser spot in the Raman measurement was $5 \mu \mathrm{m}$. The FWHM of the Raman spectra of the CLC poly-Si thin film was almost same with that of the single 
crystalline Si. But the peak position of the CLC poly-Si thin film was shifted by $4 \mathrm{~cm}^{-1}$ lower than that of the single crystalline Si. This means that the CLC poly-Si thin film has tensile strain.

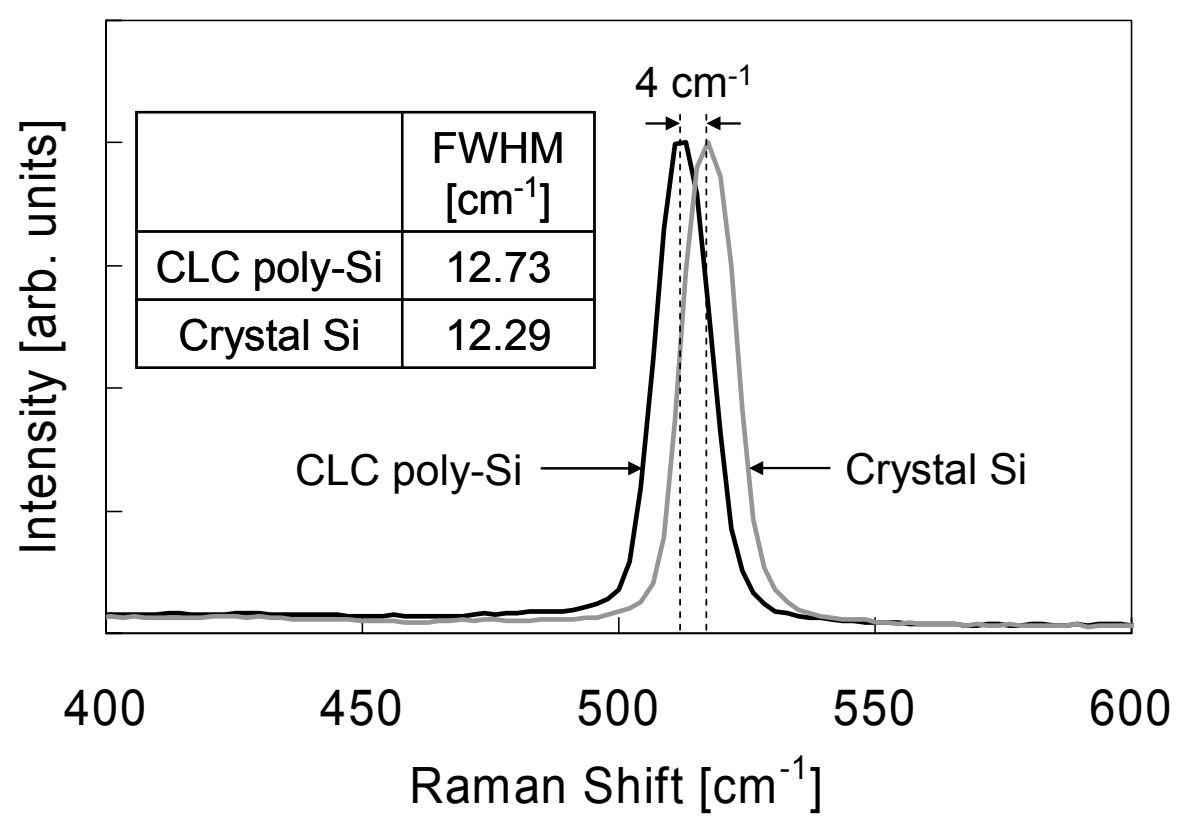

Fig. 7. Raman spectra of the CLC poly-Si thin film and single crystal Si.

Figure 8 shows XRD spectra of the CLC poly-Si thin film for the comparison of outof-plane and in-plane crystallinity. All the peaks of the in-plane spectrum were shifted to lower angles than that of the out-of-plane spectrum. The lattice constants of the CLC poly-Si thin film in out-of-plane and in-plane direction calculated from Bragg law were 5.42 and $5.46 \AA$, respectively. These values were differed from that of single crystal Si. The difference of lattice constant in in-plane direction of the CLC poly-Si thin film was $0.6 \%$ corresponding to single crystal $\mathrm{Si}$.

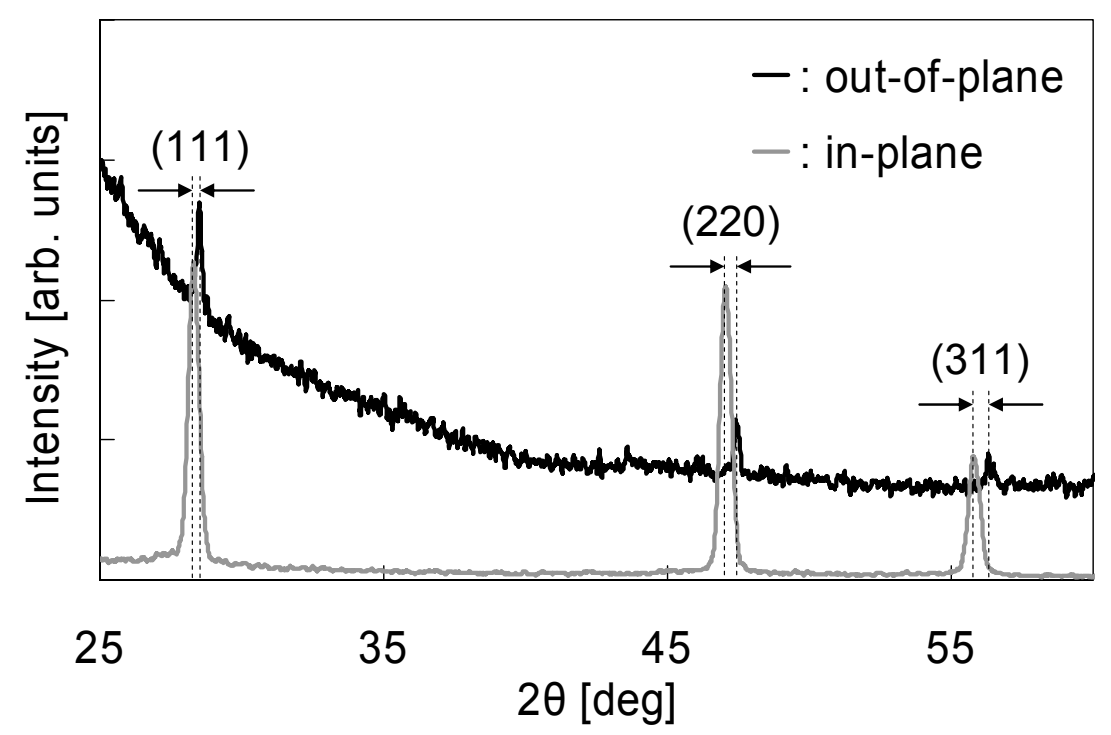

Fig. 8. XRD spectra of the CLC poly-Si thin film for the comparison of out-of-plane and in-plane crystallinity. 
This strain is estimated easily by the following equation.

$$
\xi=\left(\frac{\Delta L}{L}\right)_{S i}-\left(\frac{\Delta L}{L}\right)_{S i O_{2}}
$$

$\xi$ is the strain value and $(\Delta \mathrm{L} / \mathrm{L})$ is the shrinkage value ratio, which is defined by $\Delta \mathrm{L}=$ $\mathrm{L}$ (the melting point of $\mathrm{Si}$ ) - L(room temperature). It is simply assumed that the thermal expansion coefficients of $\mathrm{Si}$ and $\mathrm{SiO}_{2}$ are constantly $2.6 \times 10^{-6}$ and $0.5 \times 10^{-6 \circ} \mathrm{C}^{-1}(\mathrm{~T}=300$ $\mathrm{K}$ ), respectively. The calculated value of strain was $0.29 \%$, which is on the same order as that obtained by XRD measurements. Indeed, thermal expansion coefficients are changed by temperature. As temperature increases, the difference in the thermal expansion coefficients between $\mathrm{Si}$ and $\mathrm{SiO}_{2}$ is increases (9). Taking account of the temperature dependence of the thermal expansion coefficients, the difference between the calculated strain value and experimentally determined strain value becomes smaller. It is thought that the large tensile strain was induced by the large difference in thermal expansion coefficients between the Si thin film and the buffer $\mathrm{SiO}_{2}$ film.

Figure 9 shows cross-sectional TEM images of the CLC poly-Si thin film. Although it seems that there is an interfacial layer between the CLC poly-Si thin film and the buffer $\mathrm{SiO}_{2}$ film, there was not an interfacial layer as shown in Fig. 9(b). This means that large strain existed around the interface. This result supports the assumption that the strain was induced by the difference in the thermal expansion coefficients between the Si thin film and the buffer $\mathrm{SiO}_{2}$ film. White and black regions were observed in a single grain. This result shows that the strain was not uniform in a grain. It was found that the strain in CLC poly-Si thin films was not uniform locally, although the XRD and Raman spectra show that strain was uniform macroscopically.
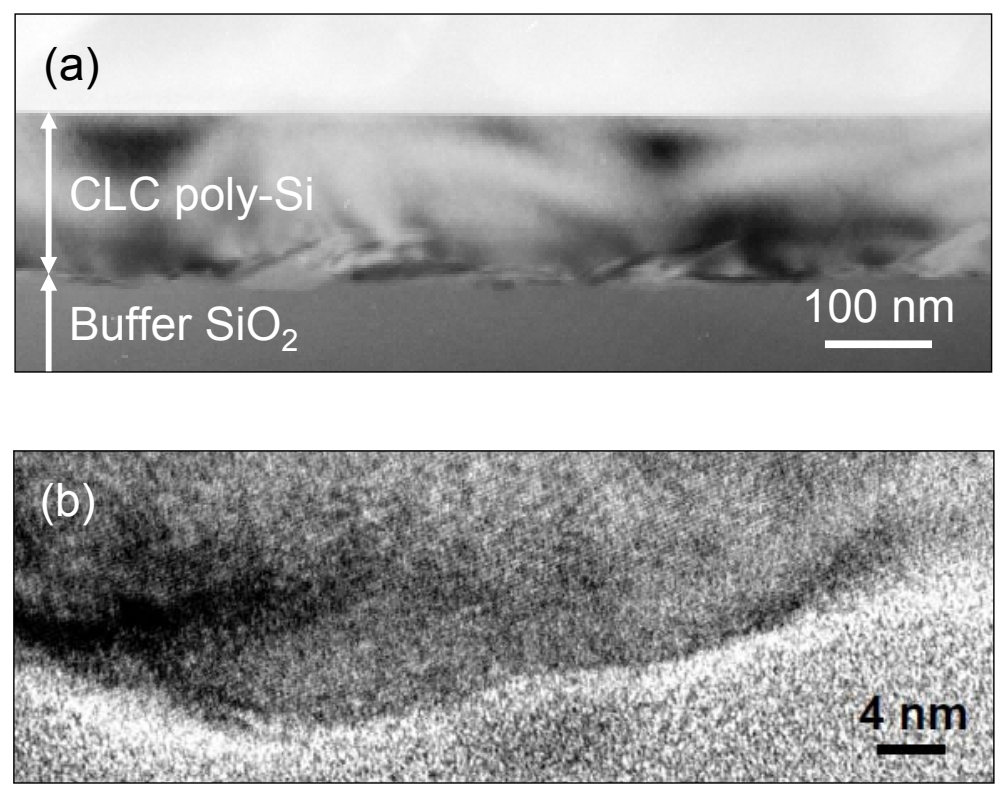

Fig. 9. Cross-sectional TEM images of the CLC poly-Si thin films. (b) is an expansion of the interface between the CLC poly-Si thin film and the buffer $\mathrm{SiO}_{2}$ film in (a). 


\section{Conclusion}

Crystallinity and internal strain of one-dimensionally long Si grains by CLC was investigated. In the CLC, Si grains with an average size of $20 \times 2 \mu \mathrm{m}^{2}$ grew along the laser scanning direction. It has been found that crystal growth of one-dimensionally long $\mathrm{Si}$ grains by CLC is performed to make stable grain boundaries. It has been also clarified that $0.6 \%$ tensile strain exists in the CLC poly-Si thin films due to the large difference in thermal expansion coefficients between $\mathrm{Si}$ thin films and the buffer $\mathrm{SiO}_{2}$ films.

\section{Acknowledgments}

A part of this work was supported by Special Coordination Fund for Promoting Science and Technology of Ministry of Education, Culture, Sports, Science and Technology (MEXT).

\section{References}

1. A. Hara, F. Takeuchi, and N. Sasaki, J. Appl. Phys. 91, 708 (2002).

2. M. Matsumura, Dig. of $12^{\text {th }}$ international Workshop on Active-Matrix LiquidCrystal Displays, 1 (2005).

3. M. A. Crowder, A. T. Voutsas, S. R. Droes, M. Moriguchi, and Y. Mitani, IEEE Trans. Electron Devices. 51, 560 (2004).

4. A. Hara, M. Takei, F. Takeuchi, K. Suga, K. Yoshino, M. Chida, T. Kakehi, Y. Ebiko, Y. Sano, and N. Sasaki, Jpn. J. Appl. Phys. 43, 1269 (2004).

5. S. Fujii, S. Kuroki, X. Zhu, M. Numata, K. Kotani, and T. Ito, Jpn. J. Appl. Phys. 47, 3046 (2008).

6. T. Sato, Y. Takahashi, H. Hara, and Y. Okamoto, Phys. Rev. B 4, 1950 (1971).

7. K. Uchida, T. Krishnamohan, K. C. Sarawat, and Y. Nishi, Tech. Dig. Int. Electron Dev. Meet., 129 (2005).

8. M. Kohyama, and R. Yamamoto, Phys. Rev. B 49, 17102 (1994).

9. H. Tada, A. E. Kumpel, R. E. Lathrop, J. B. Slanina, P. Nieva, P. Zavracky, I. N. Miaoulis, and Y. Wong, J. Appl. Phys. 87, 4189 (2000). 